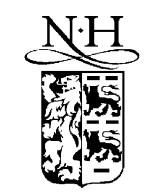

ELSEVIER

\title{
Geometric integration of conservative polynomial ODEs th
}

\author{
Robert I. McLachlan ${ }^{\mathrm{a}, *}$, G.R.W. Quispel ${ }^{\mathrm{b}}$ \\ ${ }^{a}$ Department of Mathematics, Institute of Fundamental Sciences, Massey University, Palmerston North, New Zealand \\ ${ }^{\mathrm{b}}$ Mathematics Department, LaTrobe University, Bundoora, Melbourne 3083, Australia
}

\begin{abstract}
We consider systems whose Hamiltonian is of the form $H(q, p)=\frac{1}{2} p^{2}+V(q)$, where the potential $V$ is either cubic or quartic with no cubic terms. For most of these systems (in the measure sense) we give an explicit numerical integration method that preserves both phase space volume and the value of the Hamiltonian. This is exemplified in the Hénon-Heiles system. An application is to the hybrid Monte Carlo method of statistical mechanics, where the energy preservation means that steps are never rejected, allowing the time step to be increased and the autocorrelation decreased.
\end{abstract}

๑ 2003 IMACS. Published by Elsevier Science B.V. All rights reserved.

\section{Introduction}

Many useful geometric integrators are explicit methods based on splitting and composition [4]. We consider conservative systems of ODEs. To avoid discussion of the precise nature of "conservative" systems, we immediately declare them to be those that preserve both an energy function and phase space volume. In this paper we study explicit numerical integration methods that preserve energy and phase space volume: the former provides a kind of nonlinear stability, while the latter provides Poincaré recurrence, disallows attractors, and forms the basis of ergodic theory.

A preliminary study of systems preserving volume and an integral was made in [5]. In general these systems can be written in the "skew-gradient" form

$$
\dot{x}_{i}=\sum_{j} J_{i j}(x) \frac{\partial H(x)}{\partial x_{j}},
$$

\footnotetext{
क The following paper was inadvertently omitted from the Applied Numerical Mathematics Special Issue; 'Themes in Geometric Integration' (Vol. 39, numbers 3-4, December 2001). We regret this error.

* Corresponding author.

E-mail addresses: r.mclachlan@massey.ac.nz (R.I. McLachlan), r.quispel@latrobe.edu.au (G.R.W. Quispel).
} 
where $J_{i j}=-J_{j i}, H$ is the ("energy") integral, and $\sum_{i, j} \frac{\partial J_{i j}}{\partial x_{i}} \frac{\partial H}{\partial x_{j}}=0$, implying that the vector field is divergence free and hence that Euclidean volume is preserved by its flow. Furthermore, there is much freedom in the choice of $J$ for a given system, and in many cases we can take $\sum_{i} \frac{\partial J_{i j}}{\partial x_{i}}=0$ for all $j$. This is the case in all our examples and we shall assume it from now on.

Prime examples of this case, of course, are given by Hamiltonian systems, which are naturally given in skew-gradient form with constant $J$. Although these are normally treated with symplectic integrators, there are also arguments for preserving energy instead [10]. Another application is to noncanonical ('Poisson') systems, for which Poisson integration can be extremely expensive [7]. Also, it is not obvious whether symplectic structure plays an important role in large ergodic systems; it is ignored in statistical mechanics, for example. Although the methods presented here apply to limited types of systems (classes of polynomials), energy-preserving integration is normally implicit and expensive (see, e.g., [6] and references therein), so we feel that any such special class is worth studying.

Our method is based on " $J$-splitting" [5], the only explicit energy and volume-preserving method for systems of a general form. We write $J=\sum_{j, k} J^{(j k)}$ where

$$
J_{l m}^{(j k)}= \begin{cases}J_{l m} & (l m) \in\{(j k),(k j)\}, \\ 0 & \text { otherwise, }\end{cases}
$$

to get $n(n-1) / 2$ two-dimensional systems

$$
\dot{x}=J^{(j k)}(x) \nabla H(x),
$$

in which $\dot{x}_{i}=0$ for $i \notin\{j, k\}$. Each preserves the original energy $H$. If, in addition,

$$
\frac{\partial J_{i j}}{\partial x_{i}}=0 \quad \text { for all } i, j,
$$

then each preserves Euclidean volume. Then each is area-preserving, hence formally integrable, so one numerical integration method is to evaluate and compose their flows. For example, the three-dimensional Lotka-Volterra model [8] has this form:

$$
\left(\begin{array}{c}
\dot{x} \\
\dot{y} \\
\dot{z}
\end{array}\right)=\left(\begin{array}{c}
x y-x z \\
y z-y x \\
z x-z y
\end{array}\right)=\left(\begin{array}{ccc}
0 & -1 & 1 \\
1 & 0 & -1 \\
-1 & 1 & 0
\end{array}\right) \nabla(x y z) ;
$$

the three two-dimensional flows are simple exponentials. ${ }^{1}$

For other systems, solving the two-dimensional systems (1) in closed form may be difficult. Here we consider situations in which they do not need to be solved.

Suppose $H(x)$ is a polynomial that is quadratic in $x_{i}$ and $x_{j}$ for all $i, j$ such that $J_{i j} \neq 0$. Then each system (1) is effectively linear in $x$ and can be solved in terms of elementary functions. But more is possible: we can apply the midpoint rule to (1), which preserves area, and hence (in this case) volume

${ }^{1}$ Interestingly, this $J$-splitting is identical to more conventional $H$-splitting applied to the system written in its second Hamiltonian form

$$
\left(\begin{array}{ccc}
0 & x y & -x z \\
-x y & 0 & y z \\
x z & -y z & 0
\end{array}\right) \nabla(x+y+z) .
$$

Thus it may be viewed as a Lie-Poisson, Casimir-preserving method. A similar situation holds for the free rigid body. 
in $\mathbb{R}^{n}$, and also energy, since $H$ is a quadratic function of $x$ [1]. In practice, the midpoint rule for linear systems is even easier to evaluate than the exact flow, is reversible, and is effectively explicit. (The generalised Yoshida method $[9,12]$ can subsequently be used to increase the order as desired.)

Simple mechanical systems are our main example. These are Hamiltonian with $H(q, p)=\frac{1}{2} p^{2}+$ $V(q), q, p \in \mathbb{R}^{n}$, and canonical $J=\left(\begin{array}{cc}0 & I \\ -I & 0\end{array}\right)$. Then the two-dimensional systems (1) take the form

$$
\dot{q}_{i}=p_{i}, \quad \dot{p}_{i}=-\partial V / \partial q_{i}, \quad \dot{q}_{j}=\dot{p}_{j}=0 \quad \text { for } j \neq i .
$$

For these systems the above requirement is equivalent to the condition that the potential $V(q)$ be biquadratic. (We use the word biquadratic even when $n>2$, i.e., to mean multiquadratic.) Systems with biquadratic potentials $V(q)$ are already a large class of systems which can be studied using this method. For example, the famous Hamiltonian $H=p_{1}^{2}+p_{2}^{2}+q_{1}^{2} q_{2}^{2}$, which was long thought to have energy surfaces on which the flow is ergodic, erroneously as it turns out [3], is biquadratic. When energy surfaces are unbounded (as in that case) and when $|\nabla H|$ varies widely on an energy surface, energy preservation may be important for long-time stability.

Now the biquadratic requirement is not such as a big restriction as it might appear, for one may be able to apply a linear change of variables to bring more general potentials into biquadratic form. We demonstrate below that this can be done for most (in the measure sense) cubics when $n>2$, and for many when $n=2$ - the Hénon-Heiles Hamiltonian being an example. Even for quartic potentials with no cubic part, a large set (of codimension 1 (for $n>2$ ) or 2 (for $n=2$ )) can be made biquadratic. Although expressed in terms of mechanical systems, these results also apply to any system with such a polynomial integral, provided $\partial J_{i j} / \partial x_{j}=0$ for all $i$ and $j$.

The explicit numerical integration method, where it applies, is summarised as:

(1) Write the system as $\dot{x}=J(x) \nabla H(x)$, where $H(x)$ is the first integral, $\partial J_{i j} / \partial x_{j}=0$ for all $i, j$, and $H(x)$ is quadratic in $x_{i}$ and $x_{j}$ for all $i, j$ such that $J_{i j} \neq 0$;

(2) Apply $J$-splitting to get two-dimensional linear systems;

(3) Integrate each with the midpoint rule, which is effectively explicit;

(4) Increase the order of the method by composition, if necessary.

\section{Cubic potentials}

First note that we need only examine the homogeneous cubic terms in the potential. Any quadratic terms remain quadratic after a linear change of variables, and the kinetic energy term will transform by the symplectic cotangent lift $(q, p) \mapsto(y, z), q=A y, p=A^{-\mathrm{T}} z$.

Proposition 1. The homogeneous real cubic $V(q)$ in $n$ variables can be brought into biquadratic form by a linear change of variables iff $V$ has $n$ real roots $a_{1}, \ldots, a_{n}$, linearly independent in $\mathbb{R}^{n}$. This condition holds unless $V$ takes one of the two 'bad' forms

$$
V=\left(c^{t} q\right)\left(q^{t} Q q\right)
$$

where $Q$ is a (positive or negative) definite matrix, or

$$
V=\left(c^{t} q\right)^{3}
$$

where $c \in \mathbb{R}^{n}$. The codimension of the bad cubics in the space of all cubics is $\frac{1}{6}(n-2)(n-1)(n+3)$. 
Proof. Let $V(q)=\sum_{i j k} c^{i j k} q_{i} q_{j} q_{k}$. Under the linear change of variables $q_{i}=\sum_{j} a_{i j} y_{j}, \tilde{V}(y):=$ $V(q)=\sum_{i j k l m n} c^{i j k} a_{i l} a_{j m} a_{k n} y_{l} y_{m} y_{n}$, which is biquadratic iff $\sum_{i j k} c^{i j k} a_{i l} a_{j l} a_{k l}=0$ for all $l$. But this is $V\left(a_{l}\right)$, where $a_{l}$ is the $l$ th column of the matrix $A=\left(a_{i l}\right)$. The transformation must be invertible, so any set of $n$ linearly independent roots of $V(q)=0$ makes $\widetilde{V}(y)$ biquadratic.

The set $\{q: V(q)=0\}$ is a real affine variety [2]. Because the degree of $V$ is odd, it always has dimension $n-1$. It always contains $n$ linearly independent points unless it is an $(n-1)$-plane, in which case $V$ must have a linear factor, say $c^{t} q$. For such a $V$ to be bad, the remaining quadratic factor must yield no new zeros. If it factors, this gives Eq. (4); if it does not factor, it must be a definite quadratic form, giving Eq. (3).

The space of homogeneous cubics in projective space has dimension $\left(\begin{array}{c}n+2 \\ 3\end{array}\right)-1$. The linear factors $c^{t} q$ have dimension $n-1$, and the quadratics $\frac{1}{2} n(n+1)-1$, giving the final result for the codimension of the bad set.

When $n=1$, the only cubic $V=q_{1}^{3}$ is bad.

When $n=2$, the good and bad cubics both have dimension 3. All cubics have a linear factor, while the remaining quadratic form may have eigenvalues of the same (bad) or opposite (good) sign. So we can say that roughly half of the cubics are good and half bad. The cubic part of the Hénon-Heiles potential,

$$
V=q_{1}^{2} q_{2}-\frac{1}{3} q_{2}^{3}=\frac{1}{3} q_{2}\left(3 q_{1}^{2}-q_{2}^{2}\right)=\frac{1}{3} q_{2}\left(\sqrt{3} q_{1}-q_{2}\right)\left(\sqrt{3} q_{1}+q_{2}\right),
$$

is good. For example, a convenient change of variables is generated by the roots $(1,0)$ and $(1, \sqrt{3})$. The full transformation and the resulting Hénon-Heiles Hamiltonian are

$$
\begin{aligned}
& q=\left(\begin{array}{cc}
1 & 1 \\
0 & \sqrt{3}
\end{array}\right) y, \quad p=\left(\begin{array}{cc}
1 & 0 \\
-1 / \sqrt{3} & 1 / \sqrt{3}
\end{array}\right) z, \\
& \widetilde{H}(y, z):=H(q, p)=\frac{1}{6}\left(4 z_{1}^{2}-2 z_{1} z_{2}+z_{2}^{2}\right)+\frac{1}{2} y_{1}^{2}+y_{1} y_{2}+2 y_{2}^{2}+\sqrt{3}\left(y_{1}^{2} y_{2}+2 y_{1} y_{2}^{2}\right) .
\end{aligned}
$$

After splitting, the two 2D linear systems are

$$
\left(\begin{array}{l}
\dot{y}_{1} \\
\dot{z}_{1}
\end{array}\right)=\left(\begin{array}{cc}
0 & 4 / 3 \\
-1-2 \sqrt{3} y_{2} & 0
\end{array}\right)\left(\begin{array}{l}
y_{1} \\
z_{1}
\end{array}\right)+\left(\begin{array}{c}
-z_{2} / 3 \\
-y_{2}-2 \sqrt{3} y_{2}^{2}
\end{array}\right), \quad\left(\begin{array}{l}
\dot{y}_{2} \\
\dot{z}_{2}
\end{array}\right)=\left(\begin{array}{l}
0 \\
0
\end{array}\right)
$$

and

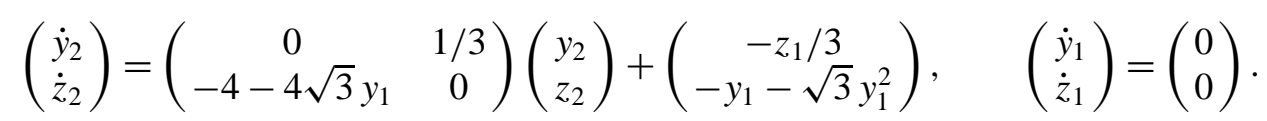

Numerical experiments with the simplest, second order method applied to the Hénon-Heiles system in this form confirm that it behaves as expected, and that volume and energy preservation lead to the persistence of invariant 2-tori in the numerics.

We also did experiments on the system with $H=p_{1}^{2}+p_{2}^{2}+q_{1}^{2} q_{2}^{2}$, interesting because all orbits are bounded but can make arbitrarily large excursions from the origin. Symplectic leapfrog always blew up when a large enough excursion happened: this occurred at $t=420,230,170$, and 1200 for time step $\Delta t=0.2,0.1,0.05$, and 0.025 , respectively, with initial conditions on the level set $H=2$. The explicit energy- and volume-preserving method, however, gave qualitatively correct results for all time and for all time steps, only degrading gradually as $\Delta t$ increased. (We tested up to $\Delta t=2$.) 
When $n=3$, the bad cubics have codimension 2 in the 9-dimensional space of cubics; when $n=4$, they have codimension 7 in the 19-dimensional space of cubics. Notice that the bad set is much larger than simply counting terms $\left(n^{2}-1\right.$ free parameters in $A$ to be used to remove only $n$ bad terms $\left.q_{i}^{3}\right)$ would suggest.

\section{Quartics}

We consider Hamiltonians with quartic but no cubic terms. As above, only the homogeneous quartic part need be considered.

Proposition 2. The homogeneous real quartic $V(q)$ in $n$ variables can be brought into biquadratic form by a linear change of variables iff $V$ has $n$ real critical points $a_{1}, \ldots, a_{n}$, linearly independent in $\mathbb{R}^{n}$. The codimension of the submanifold of the space of quartics where this condition holds is 1 for $n \neq 2$ and 2 for $n=2$.

Proof. Let $V(q)=\sum_{i j k l} c^{i j k l} q_{i} q_{j} q_{k} q_{l}$. There are $n^{2}$ 'bad' terms $y_{m}^{3} y_{p}$ and $y_{m}^{4}$ where $q=A y$. Their coefficients are

$$
\sum_{i j k l} c^{i j k l} a_{i m} a_{j m} a_{k m} a_{l p}=\nabla V\left(a_{m}\right) \cdot a_{p} .
$$

Since, for invertibility, the directions $a_{p}$ must be independent, we see that we need $\nabla V\left(a_{m}\right)=0$, $m=1, \ldots, n$. The derivatives $\partial V / \partial q_{i}$ are $n$ homogeneous cubics in $n$ variables, which do not have a simultaneous zero unless their resultant is zero [2]. For $V$ 's whose coefficients lie on the codimension one submanifold where the resultant is zero, if none of the $\partial V / \partial q_{j}$ 's factor (a generic condition when $n>2$ ), the number of zeros of $\nabla V$ in complex projective space is the Bézout number $3^{n-1}$. There are $n$ linearly independent real zeros on an open subset of this submanifold of coefficients. When $n=2$, each of the two cubics has three (possibly complex) factors, and a vanishing resultant only means that the two cubics have a factor in common. This, however, is not enough, as having two independent critical points requires two common factors, which is codimension two in the four-dimensional space of homogeneous quartics.

Here is another way to view the situation. We have $n^{2}$ bad terms to remove, and only $n^{2}-1$ parameters in $A$ to remove them with (since we operate in projective space). Thus, a codimension one 'good' set is the best we can hope for. When $n=2$, the only good quartics are $\left(b_{1} q_{1}+b_{2} q_{2}\right)^{2}\left(b_{3} q_{1}+b_{4} q_{2}\right)^{2}$, which have projective dimension 2 in the 4-dimensional space of quartics $c_{0} q_{1}^{4}+c_{1} q_{1}^{3} q_{2}+c_{2} q_{1}^{2} q_{2}^{2}+c_{3} q_{1} q_{2}^{3}+c_{4} q_{2}^{4}$. It is interesting that if the potential has no linear or quadratic terms, then the critical points used in Proposition 2 are actual critical points (equilibria) of the system. The transformation locates these at the $n$ elementary unit vectors $\left(\right.$ e.g., $\left.(1,0, \ldots, 0)^{\mathrm{T}}\right)$.

Example. Consider the family of quartics

$$
V=\frac{1}{4}\left(q_{1}^{4}+q_{2}^{4}+\alpha q_{3}^{4}\right)+\frac{1}{2}\left(a q_{1}^{2} q_{2}^{2}+b q_{2}^{2} q_{3}^{2}+c q_{3}^{2} q_{1}^{2}\right),
$$


$\alpha= \pm 1$. One factor of the resultant of $\nabla V$ is

$$
2 a b c-\left(\alpha a^{2}+b^{2}+c^{2}\right)-1
$$

which vanishes on a codimension one set of parameters, say on $a=a(b, c)$. On that set, when $\alpha=-1$ one finds that $V$ has up to 4 real lines of critical points, and for open sets of values of $b$ and $c, V$ can be put in biquadratric form. When $\alpha=1$ we must distinguish two cases. If two of $a, b, c$ are equal to -1 , then $V$ has 4 lines of real critical points and can be made biquadratic. Apart from this (codimension 2) case, there are no real nonzero critical points and $V$ cannot be put in biquadratic form.

\section{Application to the hybrid Monte Carlo method}

In the words of Sokal [11], "Correlation functions in classical equilibrium statistical mechanics or Euclidean quantum field theory are expectation values with respect to the Boltzmann-Gibbs probability measure $\mu_{\mathrm{eq}}(q)=e^{-V(q)} / \int e^{-V(q)} \mathrm{d} q$." Here $V(q)$ is the Hamiltonian of the system. (We call it $V$, not $H$, for compatibility with the previous sections.) The popular hybrid Monte Carlo (HMC) method produces samples $q^{(i)}$ by simulating the classical dynamics of the artificial Hamiltonian

$$
H(q, p)=V(q)+\frac{1}{2} p^{2} .
$$

(Other choices of $H$ are possible.) Referring the reader to [11] for details, the crucial features for us are that the update procedure $\varphi:(q, p) \rightarrow\left(q^{\prime}, p^{\prime}\right)$ must satisfy

(1) volume preservation, i.e., $\operatorname{det} d \varphi \equiv 1$;

(2) time-reversal symmetry, i.e., $R \varphi=\varphi R$ where $R:(q, p) \mapsto(q,-p)$;

(3) Metropolis acceptance, i.e., $\left(q^{\prime}, p^{\prime}\right)$ is accepted with probability $\min \left(1, \exp \left(H(q, p)-H\left(q^{\prime}, p^{\prime}\right)\right)\right)$, otherwise it is rejected.

The acceptance step ensures that, regardless of energy errors, the sample sequence has the correct probability distribution $\mu_{\mathrm{eq}}$. However, if energy errors are large, then many steps will be rejected.

The final essential criterion relates to the autocorrelation time $t_{c}$ of the samples. (This is the time, if it exists, such that the autocorrelation $\left\langle q_{n} q_{n+t}\right\rangle-\left\langle q_{n}\right\rangle^{2}=\mathcal{O}\left(e^{-t / t_{c}}\right)$ as $t \rightarrow \infty$.) Small time steps $\Delta t$ may give small energy errors and hence low rejection rates, but the samples will be highly correlated. With $n$ samples, sampling errors are $\mathcal{O}\left(\left(t_{c} / n\right)^{1 / 2}\right)$, so $n=\mathcal{O}\left(t_{c}\right)$ samples are required for a given level of error.

The most popular updating procedure is symplectic leapfrog. Higher order schemes are possible, as in symplectic integration, but have not so far proved useful. With leapfrog, to keep acceptance rates bounded means decreasing the time step as $\mathrm{Vol}^{-1 / 4}$, where $\mathrm{Vol}=L^{d}$ is the volume and $L$ the linear size of the system with dimension $d$. To stop $t_{c}$ increasing too much, samples are only taken every $\mathcal{O}(1 / \Delta t)$ time steps, at which point the momenta are 'refreshed' (replaced with new Gaussian random values).

Our primary observations are that

- it is not necessary that the update be symplectic, merely volume-preserving (for the effect of symplecticity becomes decreasingly important as $L \rightarrow \infty$ for ergodic systems); and

- it is not necessary that the update follow the classical dynamics of $H$ at all; this is only a device for staying close to the energy surface $H=$ const. 
Therefore, a volume and energy preserving update, such as the one presented here, has two enormous advantages:

- energy preservation means that steps are never rejected (the energy does not even need to be evaluated); and

- the time step can take any value, since the errors in the classical dynamics due to the finite time step are irrelevant.

This allows enormously much larger time steps, resulting in much less correlation between samples. One can also use different (e.g., large and random) time steps on each piece of $J$ in the $J$-splitting, to further decorrelate the samples. Any refinement which applies to leapfrog HMC may apply here too.

Assessing the value of this method is difficult because of the difficulty of measuring autocorrelation times accurately [11]. At present we have only tested the method on the Gaussian model with $V=$ $\sum\left(q_{i+1}-q_{i}\right)^{2}, q_{0}=-q_{n+1}$, for which $t_{c}$ can be evaluated analytically. (If the update is $q^{\prime}=A q+B p$, then $t_{c}=-1 / \ln \lambda_{\max }(A)$.) In this case we find that using $J$-splitting, and minimising $t_{c}$ with respect to the time step $\Delta t$, gives autocorrelation times very close to those using the exact flow of the Hamiltonian $H$. In such a case

- Steps are never rejected, giving a speedup factor of about 2; and

- One large $(\Delta t=\mathcal{O}(1))$ time step can be taken, giving a speedup factor of $\mathcal{O}\left(\mathrm{Vol}^{1 / 4}\right)$.

The great limitation of this method, of course, is that it only applies to special systems. It does not apply to the $\phi^{4}$ model, for example, for the potential $V(q)=\sum q_{i}^{4}$ has only the trivial critical point and hence cannot be made biquadratic. (One could still update using the exact flows of the $2 \mathrm{D}$ systems resulting from $J$-splitting; these involve inverse elliptic functions.) However, when it does apply, we essentially have the advantages of an infinite-order method with no increase in cost. The potential gains are great, so we suggest that it may be worth studying the statistical mechanics of systems with biquadratic potentials in their own right via this method.

\section{Acknowledgements}

We are grateful to Seung-Hee Joo, Hoon Hong, and Nicolas Robidoux for useful discussions, and to the Marsden Fund of the Royal Society of New Zealand and the Australian Research Council for financial support.

\section{References}

[1] G.J. Cooper, Stability of Runge-Kutta methods for trajectory problems, IMA J. Numer. Anal. 7 (1987) 1-13.

[2] D.A. Cox, J. Little, D. O’Shea, Ideals, Varieties, and Algorithms, 2nd Edition, Springer, New York, 1997.

[3] M. Gutzwiller, Chaos in Classical and Quantum Mechanics, Springer, Berlin, 1991.

[4] R.I. McLachlan, G.R.W. Quispel, Six lectures on geometric integration, in: R. DeVore, A. Iserles, E. Süli (Eds.), Foundations of Computational Mathematics, Cambridge University Press, Cambridge, 2001, pp. 155-210. 
[5] R.I. McLachlan, G.R.W. Quispel, Generating functions for dynamical systems with symmetries, integrals, and differential invariants, Phys. D 112 (1998) 298-309.

[6] R.I. McLachlan, G.R.W. Quispel, N. Robidoux, Unified approach to Hamiltonian systems, Poisson systems, gradient systems, and systems with Lyapunov functions or first integrals, Phys. Rev. Lett. 81 (1998) 2399-2403.

[7] R.I. McLachlan, C. Scovel, Equivariant constrained symplectic integration, J. Nonlinear Sci. 5 (1995) $233-256$.

[8] Y. Nakamura, T. Hashimoto, On the discretization of the three-dimensional Volterra model, Phys. Lett. A 193 (1994) 42-46.

[9] M.-Z. Qin, W.-J. Zhu, Construction of higher-order symplectic schemes by composition, Computing 27 (1992) $309-321$.

[10] J.C. Simo, N. Tarnow, The discrete energy-momentum method. Conserving algorithms for nonlinear elastodynamics, ZAMP 43 (1992) 757-792.

[11] A.D. Sokal, Bosonic algorithms, in: M. Creutz (Ed.), Quantum Fields on the Computer, World Scientific, Singapore, 1992, pp. 211-274.

[12] H. Yoshida, Construction of higher order symplectic integrators, Phys. Lett. A 150 (1990) 262-268. 